\title{
HRSP - A dedicated echelle reduction software package for Hercules
}

\author{
Jovan Skuljan \\ Department of Physics and Astronomy, University of Canterbury, \\ Private Bag 4800, Christchurch 8020, New Zealand
}

\begin{abstract}
A collection of computer programs called the Hercules Reduction Software Package (HRSP) has been developed in the Department of Physics and Astronomy, University of Canterbury, for the data reduction of échelle spectra obtained with the Hercules spectrograph. The main goal in writing a dedicated software package for Hercules was to achieve a highly-optimized reduction process that would provide the basis of a pipeline reduction performed at the Mt John University Observatory. Since the spectrograph has no moving parts, the spectrum produced in the focal plane does not move or change, so that the same reduction procedure can be applied to all CCD images. An optimized routine written especially for Hercules is always much more efficient and reliable than any general-purpose software available. HRSP is written in C and the whole reduction procedure is performed on standard FITS files. A standard échelle reduction is applied, including the background and cosmic ray subtraction, order extraction, flat-fielding, normalization and wavelength rebinning. A special feature is the radial velocity determination by crosscorrelation, which has been tested extensively over the past two years proving that Hercules is capable of delivering the high-precision radial velocities needed for detailed studying of binary stars, stellar pulsations, extra-solar planets and other related fields.
\end{abstract}

\section{Introduction}

A new échelle spectrograph called Hercules (High Efficiency and Resolution Canterbury University Large Echelle Spectrograph) has been in operation at Mt John University Observatory (MJUO) since April 2001 (Hearnshaw et al. 2002). A preliminary analysis of the radial velocities obtained with this instrument shows that a typical precision of several $\mathrm{m} \mathrm{s}^{-1}$ can be achieved (Skuljan 2003). A large number of stellar spectra has been obtained using Hercules as part of various scientific projects carried out at the University of Canterbury. Since the spectrograph has no moving parts, all images taken using the same CCD position and with the same optical fibre are practically identical. This means that there are no free parameters in the reduction process, so that it is possible to develop a dedicated, well-defined procedure which will take a set of two-dimensional raw CCD images (stellar image, thorium lamp, white lamp) and produce the corresponding one-dimensional stellar spectrum in wavelength space. As a result of this idea, the Hercules Reduction Software Package was 
written in the Department of Physics and Astronomy, University of Canterbury and is now widely used by observers working with the Hercules spectrograph.

\section{The Hercules spectrograph}

The Hercules spectrograph was designed and built at the University of Canterbury and is now in full operation at MJUO. Hercules receives light from the $\mathrm{f} / 13.5$ Cassegrain focus of the 1-m McLellan telescope. There are three optical fibres available, two of them having a diameter of $100 \mu \mathrm{m}$ (with or without a $50 \mu \mathrm{m}$ slit) and one fibre having a diameter of $50 \mu \mathrm{m}$. The corresponding resolving powers are $R=41000,70000$ and 82000 .

An R2, $200 \times 400 \mathrm{~mm}$ échelle grating provides dispersion, while a large BK7 prism in double pass is used for cross-dispersion. The wavelength coverage is designed to be $380-880 \mathrm{~nm}$ in a single exposure on a $50 \times 50 \mathrm{~mm}$ CCD. However, due to a lack of such chip, a smaller detector $(25 \times 25 \mathrm{~mm})$ is currently used. It can be mounted in one of four possible positions.

The maximum detective quantum efficiency of the fibre, spectrograph and detector system is about $18 \%$ in $2^{\prime \prime}$ seeing. High wavelength stability is achieved by installing the whole instrument in a large vacuum tank at 2-4 torr in a thermally isolated and insulated environment. The spectrograph has no moving parts, which is also an important factor for an improved stability.

\section{The Hercules Reduction Software Package}

The Hercules Reduction Software Package (HRSP) was developed at the University of Canterbury for automatic reduction of Hercules images. The package is composed of a number of individual commands designed either to perform some basic reduction steps, or to combine several simple steps into more complicated procedures. All programs are written in C under Linux. Standard FITS images are used on input (without converting them to any local image format) and the output files are also stored as FITS. This allows the user to apply any other standard software to analyse the images obtained during the reduction process.

Since most of the parameters controlling the reduction process are fixed, their values are stored in various configuration files in plain ASCII form. When all the configuration files are prepared correctly, a single HRSP command is used to reduce a given stellar spectrum. This takes about one minute of computing time and can be done at the observatory, while taking a new exposure.

\section{Reduction procedure}

Three types of CCD images are produced by the Hercules spectrograph: white lamp (for flat-fielding), thorium lamp (for wavelength calibration) and stellar spectra. The thorium images are obtained immediately before and after the stellar exposure so that the dispersion solution can be interpolated for the exact time of the stellar observation (flux-weighted mean time is used).

A typical échelle reduction is applied, including the background subtraction, cosmic ray elimination, order extraction, flat-fielding, continuum normalization 

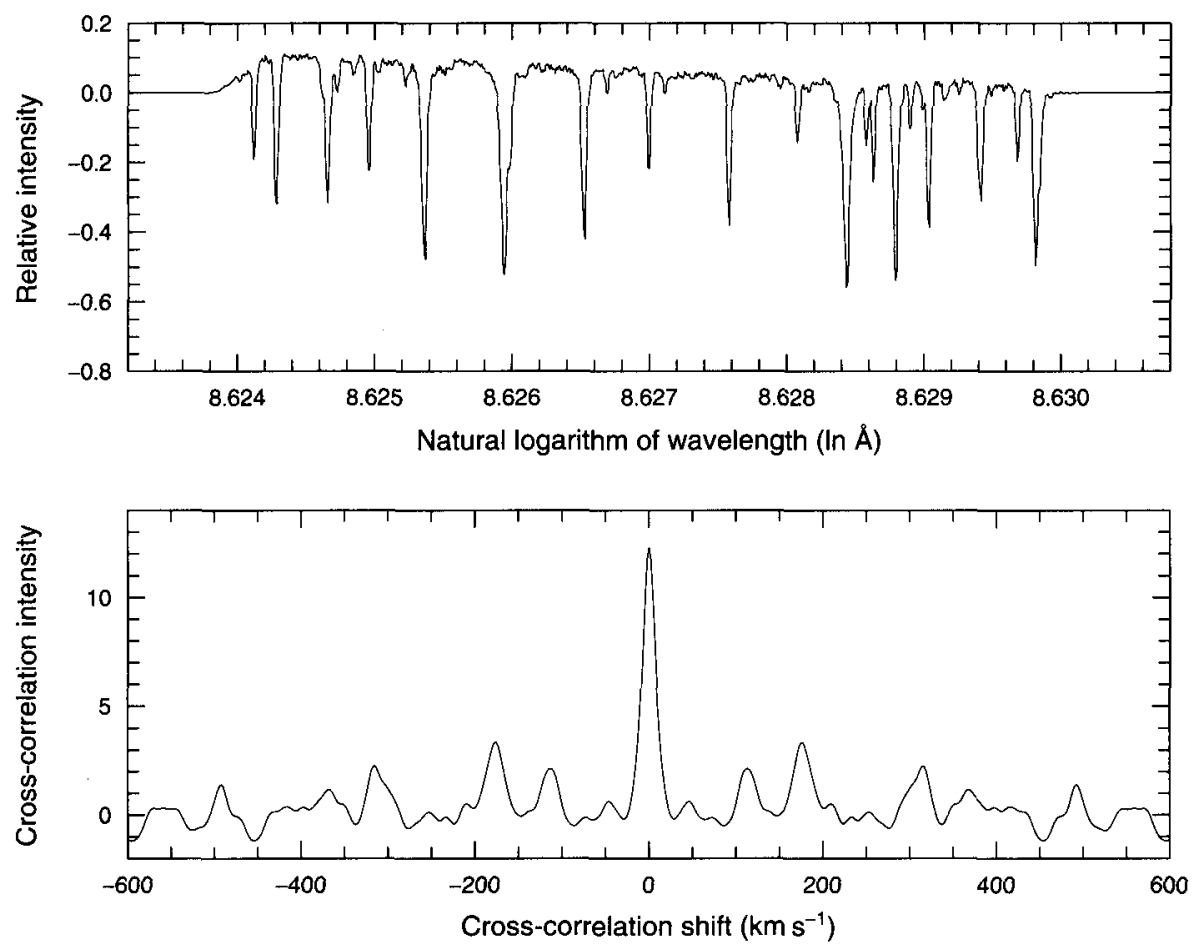

Figure 1. Top: An example of a reduced spectrum (order 102, $\mathrm{S} / \mathrm{N}$ 100) for a solar-type star, rebinned on a logarithmic wavelength scale and prepared for cross-correlation. Bottom: The corresponding cross-correlation profile.

and wavelength rebinning. The reduced stellar spectra are rebinned both on linear and logarithmic wavelength scales. The logarithmic scale is used for the determination of radial velocities by cross-correlation.

Relative stellar radial velocities are obtained by cross-correlation between two spectra of the same star. The spectra are prepared for cross-correlation by first subtracting the mean flux and then by applying a cosine-bell window to smooth out the edges (Brault \& White 1971; Simkin 1974).

A typical reduced spectrum prepared for cross-correlation and the corresponding cross-correlation profile are shown in Fig. 1.

\section{Radial velocities}

A typical long-term precision in stellar radial velocities for solar-type stars is currently about $10-15 \mathrm{~m} \mathrm{~s}^{-1}$. This can be used to search for extra-solar planets, to study stellar pulsations, or to determine very accurate orbital parameters of a spectroscopic binary star. An example is shown in Fig. 2, representing the radial velocity curve of the bright southern spectroscopic binary $\zeta \operatorname{Tr} A$, with a period 


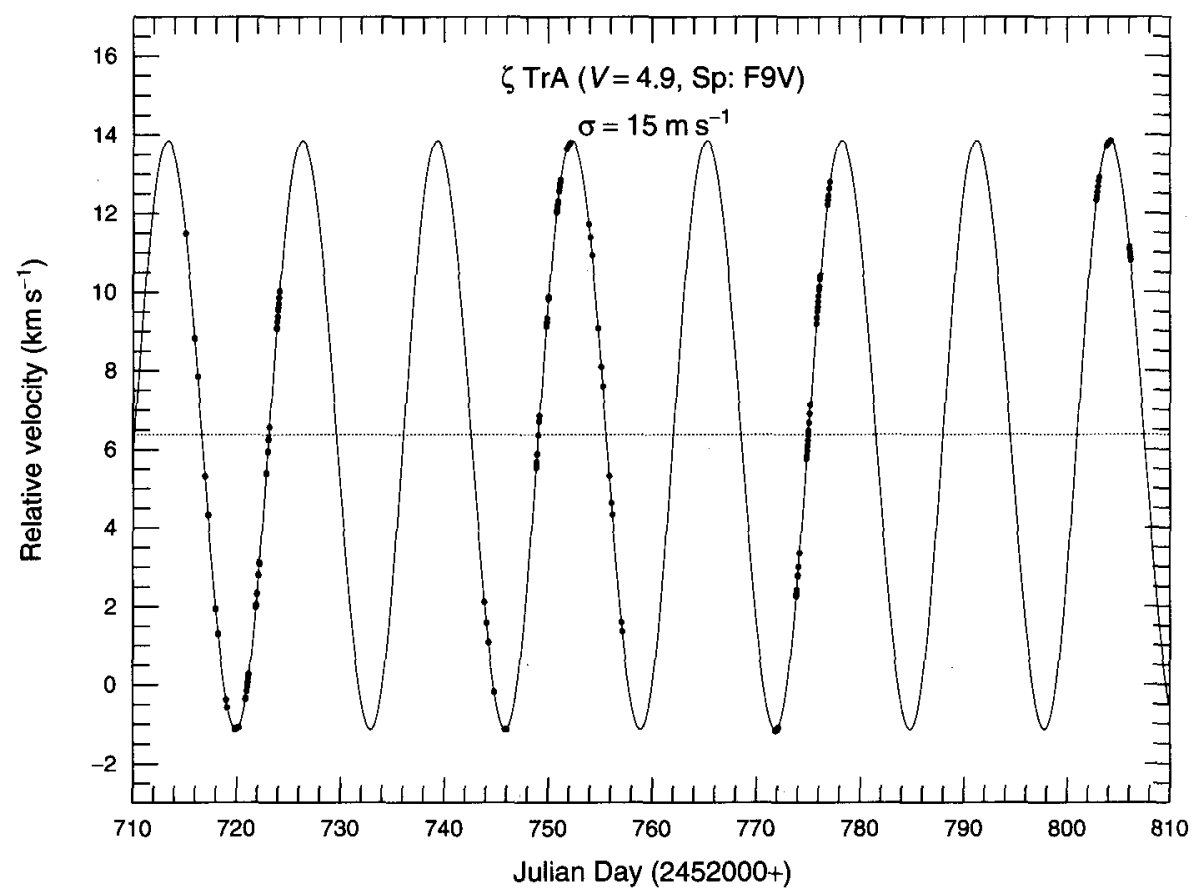

Figure 2. The radial velocity curve of $\zeta \operatorname{TrA}$. The relative velocities are measured with respect to a single observation made on JD 2452721.

of about $13 \mathrm{~d}$ and a semi-amplitude of about $7.5 \mathrm{~km} \mathrm{~s}^{-1}$. The measurements were made by Skuljan et al. (2003). The rms error of the best fit is about $15 \mathrm{~m} \mathrm{~s}^{-1}$, so that the orbital parameters can be determined with very high precision. An interesting result is that the orbit is almost circular, but still a definite ellipse, with an eccentricity of $e=0.01395 \pm 0.00022$. For a more detailed discussion on possible implications of this result see the original paper by Skuljan et al. (2003).

\section{References}

Brault, J. W., White, O.R. 1971, A\&A, 13, 169

Hearnshaw, J.B., Barnes, S.I., Kershaw, G.M., Frost, N., Graham, G., Ritchie, R., Nankivell, G.R. 2002, Experimental Astronomy, 13, 59

Simkin, S.M. 1974, A\&A, 31, 129

Skuljan, J. 2003, in ASP Conf. Ser. Vol. 289, The IAU 8th Asian-Pacific Regional Meeting, eds S. Ikeuchi, J. Hearnshaw \& T. Hanawa (San Francisco: ASP), 17

Skuljan, J., Ramm, D.J., Hearnshaw, J.B., Skuljan, L. 2003, MNRAS, submitted 